\title{
Simple optical setup for the undergraduate experimental measurement of the refractive indices and attenuation coefficient of liquid samples and characterization of laser beam profile
}

\section{Dipankar Sengupta, Bora Ung}

Dipankar Sengupta, Bora Ung, "Simple optical setup for the undergraduate experimental measurement of the refractive indices and attenuation coefficient of liquid samples and characterization of laser beam profile," Proc. SPIE 11143, Fifteenth Conference on Education and Training in Optics and Photonics: ETOP 2019, 1114319 (2 July 2019); doi: 10.1117/12.2523831 


\title{
Simple optical setup for the undergraduate experimental measurement of the refractive indices and attenuation coefficient of liquid samples and characterization of laser beam profile
}

\author{
Dipankar Sengupta, Bora Ung* \\ École de technologie supérieure, 1100 Notre-Dame St W, Montreal, QC, Canada, H3C 1K3 \\ *bora.ung@etsmtl.ca
}

\begin{abstract}
An optical setup was devised for the Electrical engineering undergraduate course "Photonic devices" where students were introduced to a simple visible diode laser based method of measuring the refractive indices of liquid samples in a transparent quartz cuvette placed on a computer-controlled rotating stage. When setting the cuvette at a small angle with respect to the incident laser beam, the light transmission through the cuvette results in a small mm-scale deflection of the laser path. The evaluation of the sample's refractive index hinges on measuring the beam displacement. Moreover, by positioning the cuvette at normal incidence and recording the optical power after passing through various liquid samples (e.g. distilled water, maple syrup) and comparing with a reference (empty cuvette), students learn to estimate the attenuation coefficients of the substances by taking into account the contribution of the multiple Fresnel reflections. Finally, the same optical setup is also used by students to perform the "knife-edge" technique for the characterization of the beam profile emitted by the visible diode laser used in the setup. The proposed setup was implemented in the fall 2018 where undergraduate students were able to practice optical alignment and implement the concepts of material refractive indices and attenuation, as well as revisit the Gaussian beam theory taught in-class.
\end{abstract}

Keywords: Angle of refraction, refractive index, optical absorption, laser beam, knife-edge method,

\section{INTRODUCTION}

Students enrolled in the later stages (typically $3^{\text {rd }}$ year onward) of the electrical engineering bachelor degree at ETS can elect to take an introductory course in the field of photonics (ELE771-Photonic devices) that has a strong emphasis on the practical aspects of lightwave technologies. One way to achieve this goal is through 8 on-hands laboratory sessions involving basic optical and photonic systems, student manipulations and measurements that are ultimately evaluated via written technical reports submitted to the instructor. Some of the basic physical topics introduced in this course relate to optical refraction, light polarization, optical attenuation, free-space and guidedoptic propagation, along with optical cavities, laser theory, optical amplifiers and photodetectors. Class lectures consist in the standard presentation of the theory and description of related examples following a textbook [1]. Lab sessions represent opportunities for student to firstly, get excited by photonic technologies (this is an unofficial mission of this course!) through on-hands operation of advanced instruments, the precise manipulation 
of delicate components and the creation/characterization of photonic systems; and secondly, to complement the physical theory seen in class through student experimentation.

The first step in this lab experiment is the characterization of the laser beam diameter. A laboratory procedure has been created for students to explore laser beam diameter using knife-edge method [2] through a pre-assembled optical setup on a mini optical breadboard that allow them to learn basic optical alignment. In brief, students are invited to measure the lateral beam displacement while passing through different types of liquids and extract from these optical measurements the corresponding values of the refractive index of the liquids. By comparing their experimental measurements with published scientific data, students must interpret the observed discrepancies through proper identification of the sources of errors and their relative importance. The students enrolled in the course follow the written lab procedures to perform while enjoying support by the technician and laboratory instructor.

\section{THEORY}

The value of the refractive index (RI) of solid and liquid substances provides key information regarding the optical, mechanical and chemical properties of samples. For example, some biomedical instruments measure changes in the refractive index in a solution to monitor the antigen-antibody reactions for pharmaceutical and medical applications. In this laboratory, undergraduate students are invited to experimentally measure the RIs of distilled water and a sample of maple syrup through their interaction with laser light. These measurements can be used, among other things, to evaluate the purity of the samples involved.

The experimental setup for measuring the refractive indices (RI) of these liquids is based on a scheme published by S. Nemoto [2], the diagram of which is shown in Fig. 1.

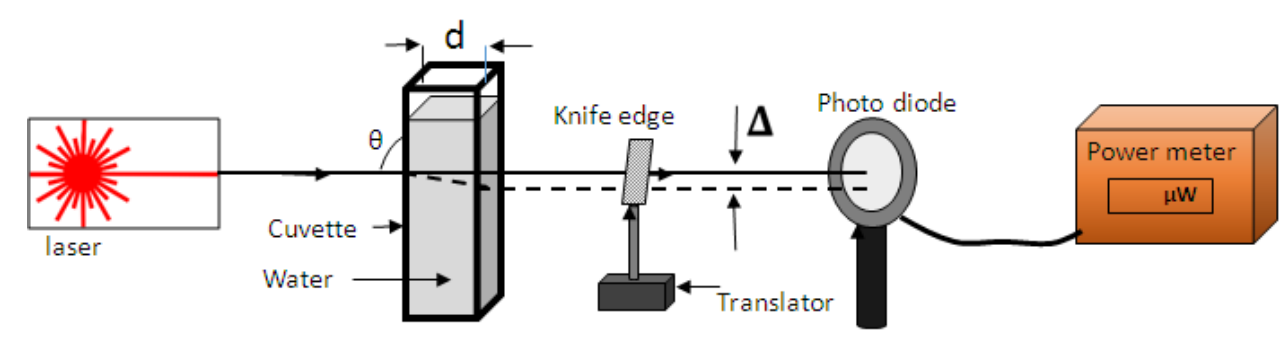

Fig. 1: Experimental setup for measuring the refractive index of a liquid

In this scheme, the transverse displacement $(\Delta)$ of a laser beam passing through a cuvette filled with a partially transparent liquid is related to the value of the liquid's refractive index $\left(n_{2}\right)$ by the following formula [2]:

$$
n_{2}=n_{0} \sin \theta \sqrt{1+\left[\frac{\cos \theta}{\sin \theta-\Delta / d_{2}}\right]^{2}}
$$

where $\theta$ represents the angle of incidence of the laser beam with respect to the input face of the cuvette, $n_{0}=1$ is the index of refraction of the external environment (air), and $d_{2}=1 \mathrm{~cm}$ is the internal dimension of the cuvette. 
Moreover, it is recalled that the reflectivity $(R)$ and transmittance $(T)$ at normal incidence of an interface between two media of dissimilar RIs ( $n_{1}$ and $n_{2}$ ) is given by the following Fresnel relations:

$$
R=\left|\frac{n_{1}-n_{2}}{n_{1}+n_{2}}\right|^{2} \text { and } T=1-R
$$

The attenuation coefficient $(\alpha)$, often measured in units of $\mathrm{m}^{-1} \mathrm{or}^{-1}$, in the propagation of a light beam through a lossy medium of length $L$ is defined by the expression:

$$
\alpha=\frac{1}{L} \ln \left(\frac{P_{i}}{P_{o}}\right)
$$

Where, $P_{\mathrm{i}}$ and $P_{\mathrm{o}}$ respectively denote the measured optical powers (in Watts) at the input and output facets of the absorbing medium.

\section{EXPERIMENT AND ANALYSIS}

\section{LIST OF PARTS}

1. $532 \mathrm{~nm}$ diode laser, Class II (CPS532-C2, Thorlabs)

2. Rotating stage (ELL8/M, Thorlabs)

3. Quartz cuvette, $3500 \mu \mathrm{L}$ (CV10Q3500S, Thorlabs)

4. Knife edge (Digital Vernier caliper)

5. 12" x 18" Breadboard (MB1218, Thorlabs)

6. Iris (ID12, Thorlabs)

7. Photo detector (S120C, Thorlabs)

8. Digital rotational stage (ELL8/M, Thorlabs)

9. Power supply for laser diode (LDS5, Thorlabs)

10. USB Power meter (PM100 USB, Thorlabs) with computer connected.

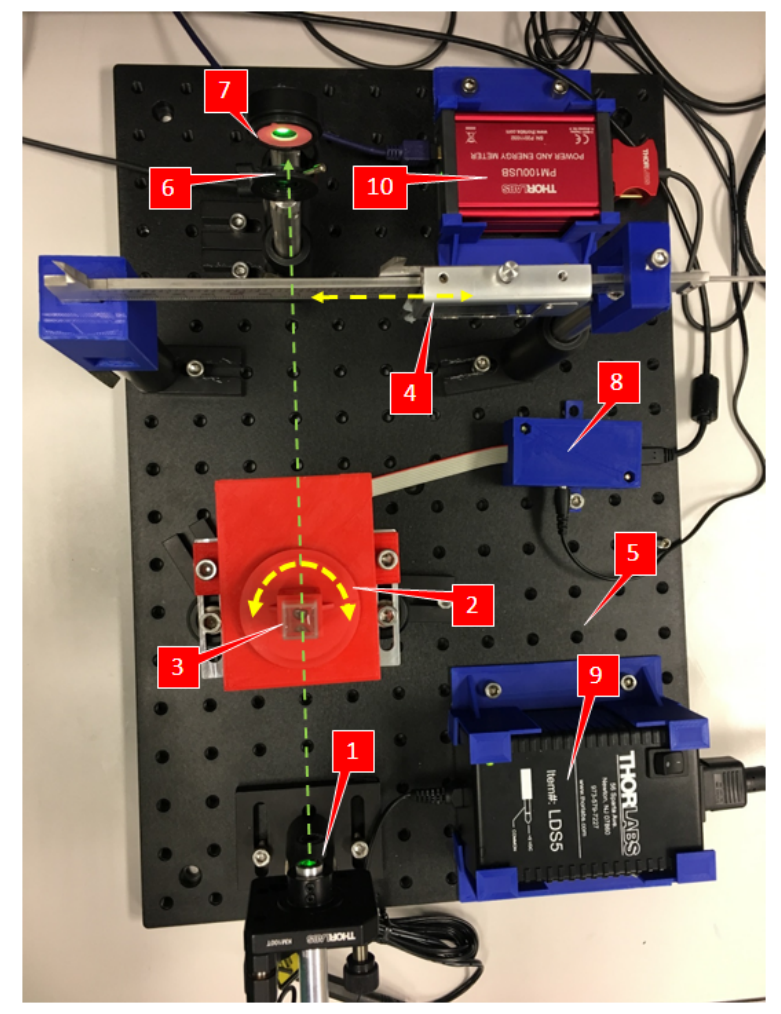

Fig. 2. Image of the experimental assembly with parts numbered

The image of the experimental setup is shown in Fig. 2. The whole lab session is divided into four parts.

A) First part (15 min): characterization of the Gaussian laser beam profile via the knife-edge method 
A common way to measure the cross-sectional profile of a Gaussian beam is by passing a razor blade through the incident Gaussian beam, and measuring the power as a function of the transverse position $(x)$ of the blade [Fig. $3 a]$. As the blade is gradually removed from the optical path of the beam, we record the power measured at the photodiode $\left(P_{N}\right)$ as a function of the transverse position $(x)$ of the blade. The resulting unit-normalized curve [Fig. $3 b]$ can be approximately modeled with an analytical expression containing an error function:

$$
P_{N}(x)=\frac{1}{2}\left[1+\operatorname{erf}\left(\frac{x-x_{0}}{w}\right)\right]
$$

It is interesting to note that the derivative of the unit-normalized power with respect to $x$ gives the following expression, which describes a Gaussian beam profile of radius $w$ :

$$
\frac{d P_{N}(x)}{d x}=\frac{1}{w \sqrt{\pi}} \exp \left[-\left(\frac{x-x_{0}}{w}\right)^{2}\right]
$$

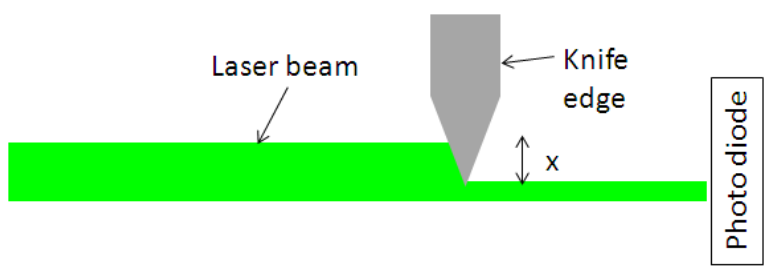

Fig 3 (a)

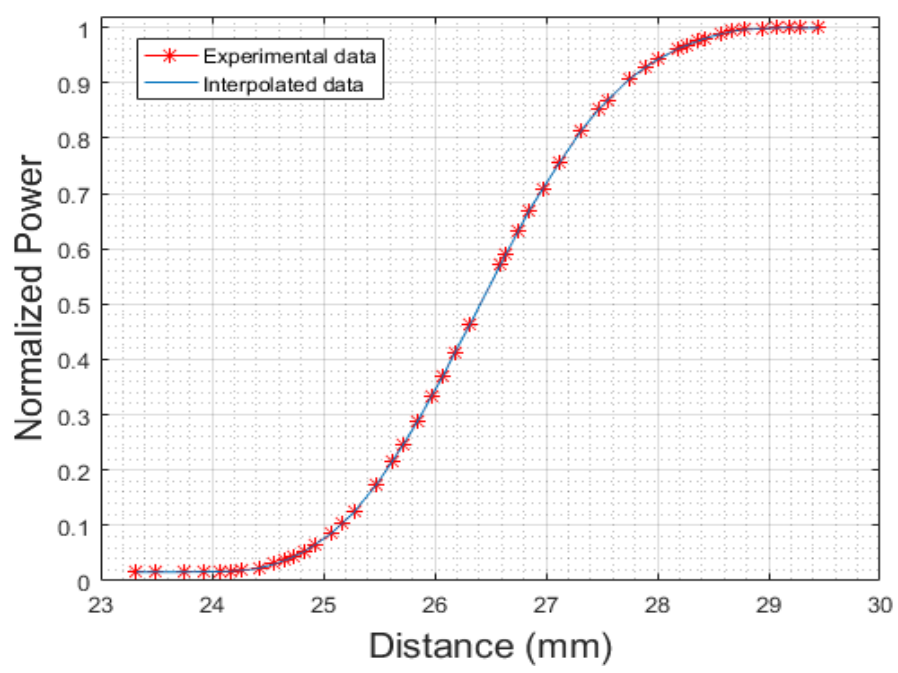

Fig 3 (b)

Figure 3 (a) Schematic of the knife-edge method. (b) Example of recorded optical power as a function of the position $(x)$ of the knife edge.

Therefore in order to characterize the laser beam profile, students need to use the optical experimental setup depicted in Fig. 2, albeit with the cuvette removed, and record the optical power of ambient light in the absence of laser. Then switch $\mathrm{ON}$ the laser and block the laser beam from illuminating the photodetector using the movable jaws of a Vernier caliper. In order to enhance the precision of the measurements, a sharp blade was hot-glued on the movable jaw. Subsequently, the blade of the Vernier is gradually translated with $0.1 \mathrm{~mm}$ steps until the blade enables the beam to pass completely such that the recorded power is maximum and stable. The derivative of the measurements in Fig. 3(b) produces the expected Gaussian profile in Fig. 4, from which the beam's FWHM and radius can be extracted by the students [Table 1]. 


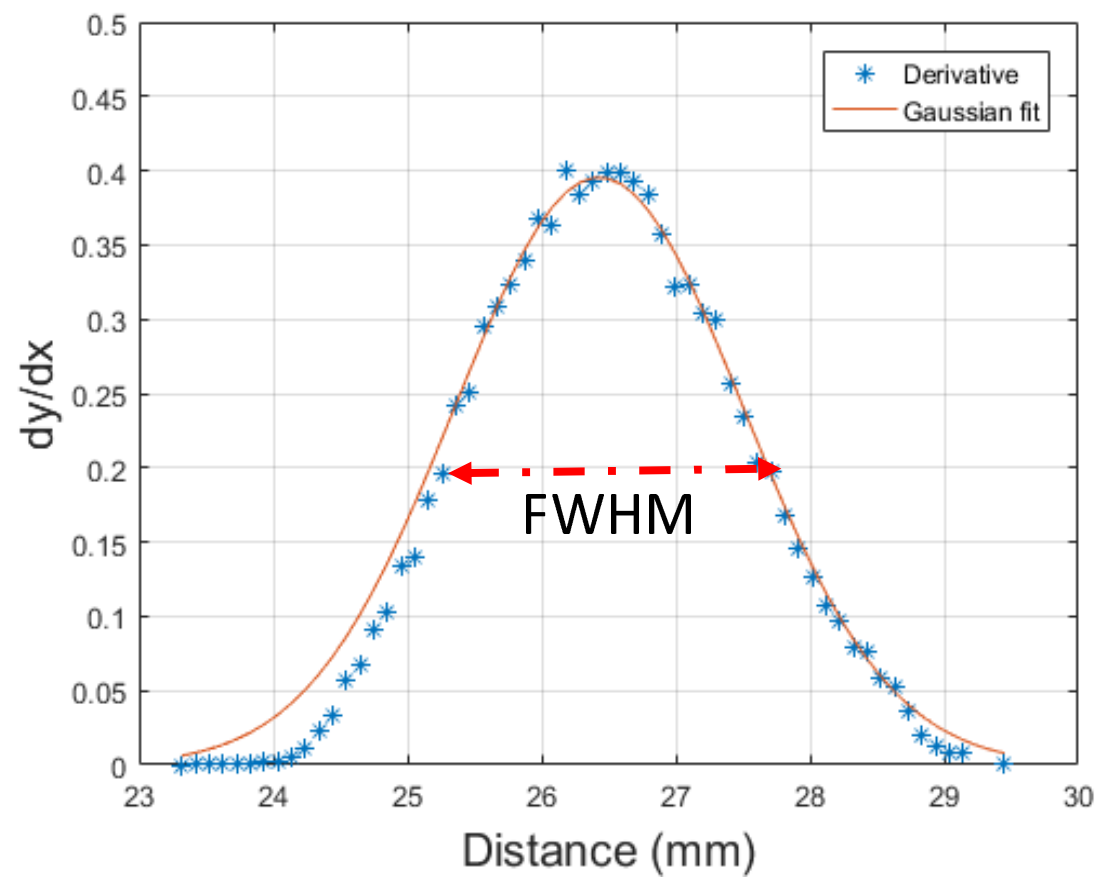

Fig. 4 Laser beam profile obtained through the knife-edge method

Table 1

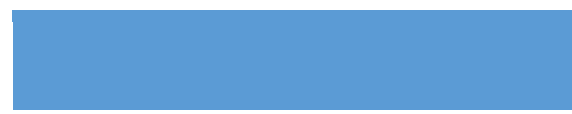

\section{Experimental}

Spec Sheet

Laser power

$0.935 \mathrm{~mW}$

$0.9 \mathrm{~mW}$

FWHM

$2.4 \mathrm{~mm}$

$3.5 \mathrm{~mm}$

The students are then asked to compare their experimental measurement of the beam's FWHM with the beam diameter value indicated on the manufacturer's specs sheet and discuss any discrepancies observed. Here it is important to note that the manufacturer indicates a slightly larger beam diameter (not FWHM) since the beam is not perfectly Gaussian but instead "circular" (because the laser is multimoded). 
(B) Second part (10 min): precise setting of the zero reference angle.

In order for the measurements of the refractive indices (RIs) of different liquids to be precise, it is critical to properly calibrate the angular position $\theta$ of the cuvette relative to the incident laser beam.

At the beginning of the lab session, students are invited to turn ON the laser diode so as to allow it to stabilize after a few minutes. Measure the input power of laser $\left(P_{\text {in }}\right)$. Carefully fill the cuvette up to $3 / 4$ with distilled water while taking care to not create air bubbles to not dirty the clear facets of the cuvette. Then place the cuvette on its support holder (custom-fabricated via 3D printing). Turn on the software of the digitally controlled rotating platform. Adjust the angle of the cuvette to about $\theta=-10^{\circ}$ (with respect to normal incidence [Fig. 5]). Perform a coarse angular sweep from $-10^{\circ}$ to $+10^{\circ}$ (with steps of $1^{\circ}$ ) and note the value of the angle $\theta_{\max }$ where the measured power is maximum. Perfect normal incidence should occur at $\theta=0^{\circ}=\theta_{\max }$, i.e. the reference angle.

To obtain a more accurate value of the reference angle, perform a finer angular sweep about $+/-2^{\circ}$ of the value $\theta_{\max }$ with steps size of $0.2^{\circ}$ and record the measured optical power. Present the results on a graph of power as a function of angle $\theta$ and identify on this graph the precise value of $\theta_{\max }$. Position the platform at $\theta=\theta_{\max }$ and set in the control software reference "zero" to this position. Remove the water from the cuvette with a pipette, dry the cuvette with some jets of compressed air and place it back on the setup. Finally, measure the transmitted power through the empty cuvette at $\theta=\theta_{\max }$ (this will be used in part $\mathrm{D}$ ).

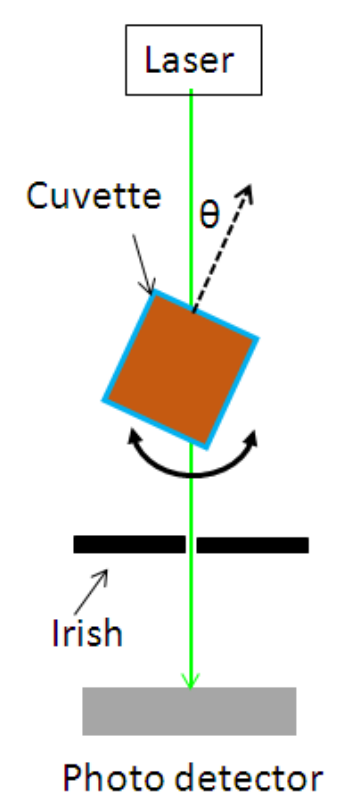

Fig. 5. Zero reference angle setting

\section{(C) Third part (45 min): Measurement of refractive index values of common liquids}

Rotate the empty cuvette to the angle $\theta=10^{\circ}$. Move the blade of the Vernier so that it completely blocks the beam (min power). Gradually translate the blade of the Vernier with steps of $0.1 \mathrm{~mm}$ until the blade completely unblocks the beam while recording the optical power measurements (as a function of blade distance) in a spreadsheet file. Fill the cuvette to $3 / 4$ with distilled water. Then repeat the same procedure as with empty cuvette (with measurements recorded in the same spreadsheet file). Remove water from the cuvette, dry and fill it with maple syrup up to $3 / 4$. Repeat the same procedure with the maple syrup. Plot the recorded data in absolute power values as in Fig. 6(a). The next step is to normalize the measurements (to unit max power) and present the results as a function of the blade position $(x)$ for the three cases: empty cuvette (air), distilled water and maple syrup [Fig. 6(b).

The value of the transverse beam displacement $(\Delta)$, which has a direct relationship with the RI values of water and maple syrup, is calculated with respect to the reference curve (A) corresponding to air (i.e. empty cuvette) in Table 2. Then using Eq.(1) students can easily retrieve the RI values of distilled water and maple syrup. 
We first ask students to compare the calculated RI of distilled water with the RI value of water presented in a public database based on published scientific data [3]. In most cases the experimentally measured value of water RI will be inaccurate ( $>3 \%$ error). Students must then explain their results, suggest what are the main possible causes of errors, and indicate the two most important causes of errors in their opinion by providing some supporting arguments. The three most important causes are: 1) the error in setting the reference zero angle $\theta_{\max }$ (because the resolution of the piezoelectric rotating stage is $+/-0.01^{\circ}$ and also due to manipulation mistakes in part $\mathrm{B}$ of the lab protocol); 2) random fluctuations in ambient lighting due to people moving near the optical setup which introduces some noise in the measurements performed in parts $\mathrm{B}$ and $\mathrm{C}$ of the protocol, and 3) the use of a laser source with low polarization extinction ratio $(5 \mathrm{~dB}$ typical) such that light is partially polarized thus allowing polarization-dependent effects to occur.

The most curious and enterprising students will rightly attempt to correct for the inaccurate setting of the $\theta_{\max }$ reference angle. This correction can be done by inserting the correct published RI value of distilled water in Eq.(1) and deducing the "real" angle $\theta$ ' at which the cuvette is set. An example of such corrected angle $\left(8.26^{\circ}\right)$ is shown in Table 3. Using the same corrected angle, students can then find a more realistic value for the RI of maple syrup (RI around 1.4634 depending on its exact biochemical composition: glucose-fructose concentration, impurities, etc.) as shown in Table 3.

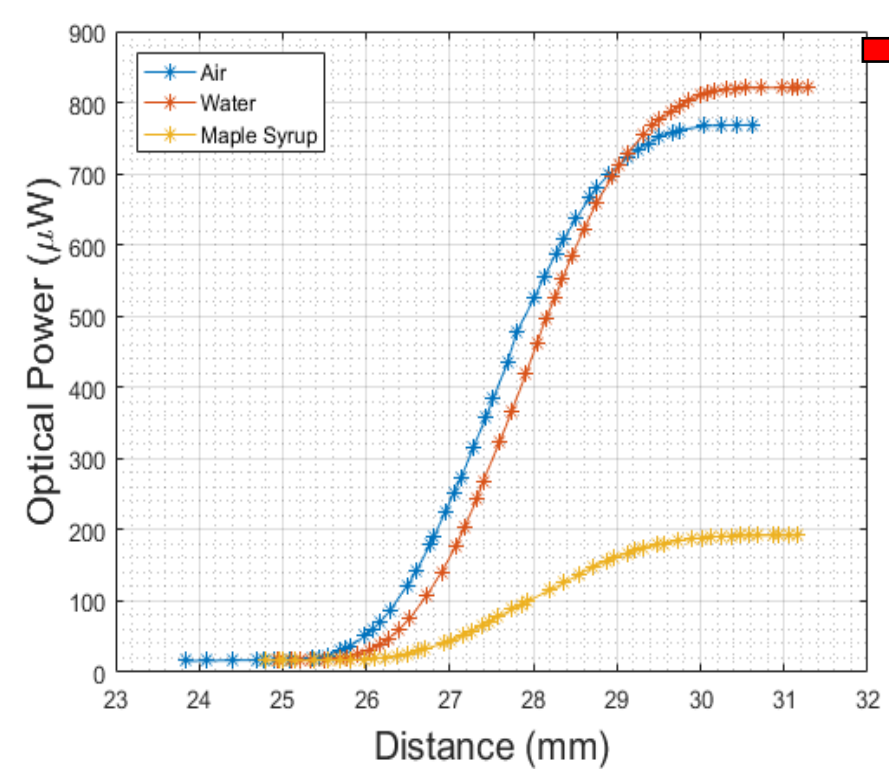

Fig. 6(a) Optical power vs. knife edge position

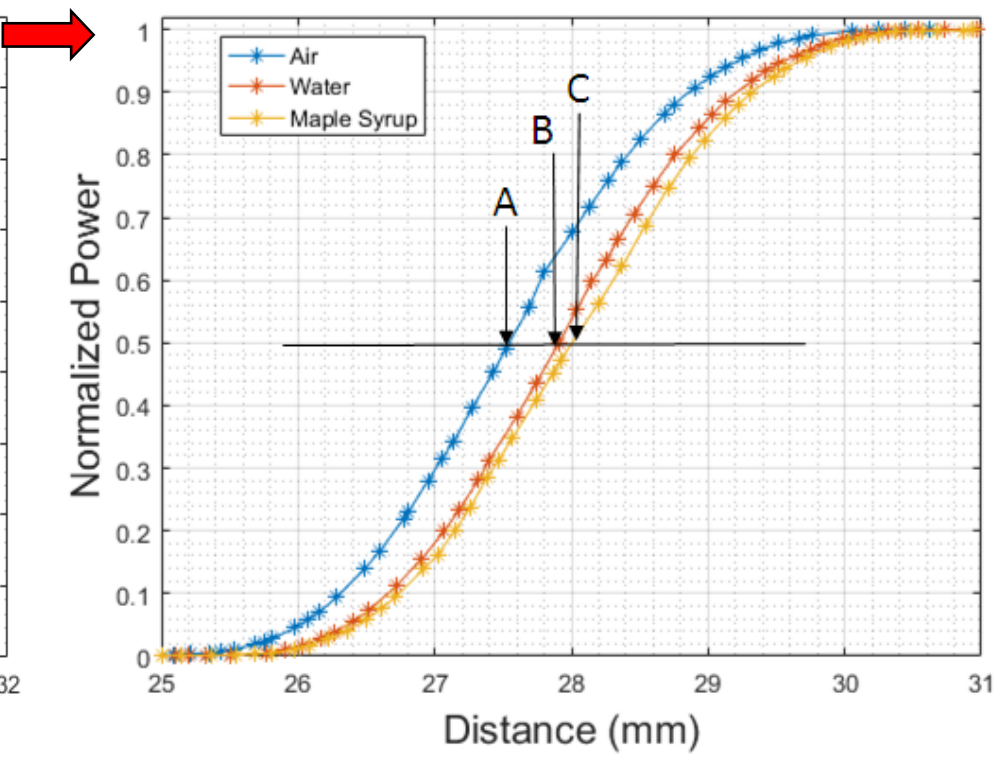

Fig. 6(b) Normalized optical power vs. knife edge position

Table 2

\begin{tabular}{|l|l|l|l|}
\hline & Air (A) & Water(B) & Maple (C) \\
\hline $\begin{array}{l}\text { Distance }(\mathrm{mm}) \text { at } \\
\text { Half power point } \\
\text { (from the graph) }\end{array}$ & 27.5451 & 27.9097 & 28.0054 \\
\hline
\end{tabular}


Table 3

\begin{tabular}{|l|l|l|l|l|l|}
\hline Angle $\theta$ & Experimental RI value & \multicolumn{3}{|c|}{ Correction } \\
\hline & Water & $\begin{array}{l}\text { Maple } \\
\text { syrup }\end{array}$ & $\begin{array}{l}\text { Correct RI value of } \\
\text { Water (literature } \\
@ 532 \mathrm{~nm})\end{array}$ & $\begin{array}{l}\text { Correct value } \\
\text { of } \theta\end{array}$ & $\begin{array}{l}\text { Corrected RI value } \\
\text { of Maple syrup }\end{array}$ \\
\hline $10^{\circ}$ & 1.2586 & 1.3513 & 1,3337 & $8,26^{\circ}$ & 1,4634 \\
\hline
\end{tabular}

(D) Fourth part (10 min): Calculation of the optical absorption coefficients of water and maple syrup

Using the same simple optical setup [Fig. 2] with the cuvette set at normal incidence $(\theta=0)$ and the blade completely out of the beam's way, it is possible to obtain a rough estimate of the light attenuation coefficients at $\lambda$ $=532 \mathrm{~nm}$ in distilled water and maple syrup. Students must take into account that the laser beam passes through 4 dielectric interfaces before reaching the photodetector [see Fig.7].

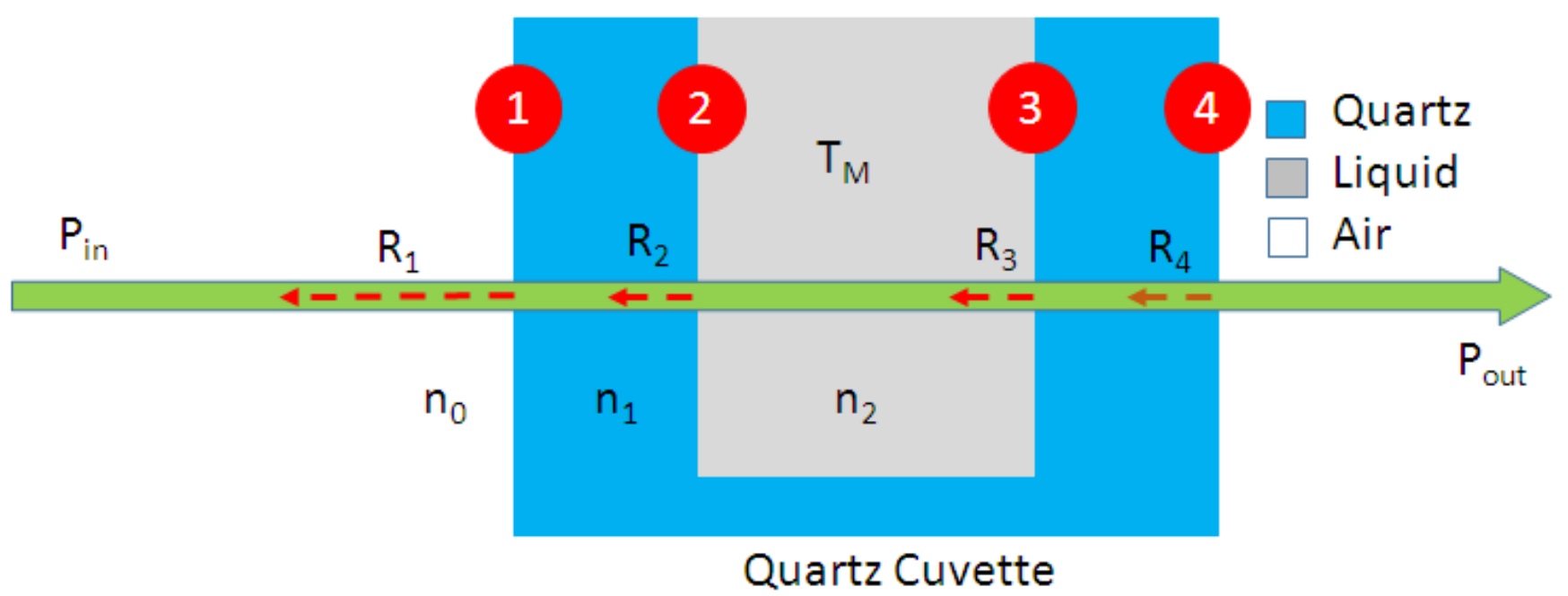

Fig. 7 Scheme for the measurement of liquid samples attenuation coefficients

where $n_{0}$ : refractive index of air ; $n_{1}:$ refractive index of quartz; $n_{2}:$ refractive index of sample ; $1,2,3$ and 4 denote the interfaces between two medium; $T_{\mathrm{M}}$ : Transmittance of the sample medium inside the cuvette. 
The laser beam enters the cuvette from the left in Fig. 7 with incident power $P_{\text {in }}$ and exits the cuvette with a lower power $\left(P_{\text {out }}\right)$ due to Fresnel losses at the various dielectric interfaces and because of optical absorption in the liquid sample, as described by the following expression:

$$
P_{\text {out }}=T_{1} T_{2} T_{3} T_{4} T_{M} P_{\text {in }}
$$

where $T_{1}, \ldots, T_{4}$ are the transmittance values at normal incidence given by $T_{i}=1-\left(n_{i}-n_{j}\right)^{2} /\left(n_{i}+n_{j}\right)^{2}$ of the corresponding dielectric interfaces $i=1,2, \ldots 4$, and $T_{M}$ denotes the transmittance of the sample medium inside the cuvette. The first step in this procedure is to find the value of $P_{\text {in }}$. This is done by measuring $P_{\text {out }}$ with an empty cuvette (with negligible absorption $T_{M}=1$ and $n_{2}=1$ ) at normal incidence. In principle, this measurement was already done in Part B, but students can redo it if deemed necessary. Knowing that the RI of quartz at $\lambda=532 \mathrm{~nm}$ is $n=1.4607$ [3], students are then instructed to retrieve the unknown incident power value by isolating $P_{\text {in }}$ in Eq.(6).

The second step involves inserting the liquid sample (water first then maple syrup, in order to lower risks of cross-contamination) and then repeat the measurement of $P_{\text {out }}$ at normal incidence. Since the value of $P_{\text {in }}$, was found in the previous step, students can now retrieve the unknown transmittance of the liquid sample by isolating $T_{\mathrm{M}}$ in Eq.(6) and subsequently deduce the sample's attenuation coefficient ( $\alpha$ in $\mathrm{cm}^{-1} \mathrm{or} \mathrm{m}^{-1}$ ) through the relation:

$$
\alpha=\frac{1}{L} \ln \left(\frac{1}{T_{M}}\right)
$$

Students must describe the logical procedure that they used as well as the calculations performed and present their results in a table similar to Table 4 . They are also instructed to compare their measurements of the attenuation coefficient in water with a published value [3] of $\alpha_{\text {water }}=3.16 \times 10^{-4} \mathrm{~cm}^{-1}$ at the laser wavelength $(532 \mathrm{~nm})$. We expect significant deviations between the experimental and published values, as shown in the exemplar data in Table 4. Students must discuss the potential sources of errors that could explain these discrepancies: temporal fluctuations in laser power, light scattering on air bubbles or impurities, bad cuvette cleaning leading to sample contamination, noise due to ambient lighting, etc. Moreover, students are asked to comment on how realistic in their view is the measured value for the attenuation coefficient of maple syrup.

\begin{tabular}{|c|c|c|c|c|}
\hline Liquid & $\begin{array}{l}\text { Refractive } \\
\text { index at } 532 \mathrm{~nm}\end{array}$ & $\mathbf{P}_{\text {out }}(\boldsymbol{\mu W})$ & Attenuation coefficient $\left(\mathrm{cm}^{-1}\right)$ & Referencevalue@532nm \\
\hline Water & $1.3337 \quad[3]$ & 829.3 & 0.04487 & $3.16 \times 10^{-4} \mathrm{~cm}^{-1}$ \\
\hline $\begin{array}{l}\text { Maple } \\
\text { syrup }\end{array}$ & 1.4634 & 193 & 1.5068 & NA \\
\hline
\end{tabular}

Table 4 


\section{CONCLUSION}

In this paper we have presented an undergraduate laboratory experiment involving the measurement of the refractive index and attenuation coefficient of common liquid samples. In a single lab session (around 90 minutes), students apply concepts in light refraction, transmission and reflexion (via application of Fresnel relations and Snell's law), Gaussian beam physics and optical propagation in lossy dielectric media. The students are also introduced to basics in optical alignment with free-space optics, laser beam profiling via the knife-edge method, and biochemical optical analysis by means of a simple setup conveniently controlled via a graphical user interface on a computer. One of the main objectives of this lab session is to initiate and excite students to optics and photonics with a hands-on approach to learning and encourage them to hone their critical thinking. In that regard, given the limited time to accomplish all the requested procedures once, students were not expected to produce experimental data that perfectly matches results found in the published literature. Instead, students were encouraged to discuss their results along the scientific method, to identify the potential sources of errors in their experiment and to learn the importance of precise manipulations, setup calibration and preparation during optical experiments.

\section{ACKNOWLEDGEMENT}

The authors are grateful to the technicians and staff engineers, André Zalzal and Samuel Gagné, of the Electrical engineering department at ETS, Montreal for their help in devising the 3D-printed components for the setup and the assembly and control of opto-electronic components.

\section{REFERENCES}

[1] S.O. Kasap, Optoelectronics and photonics Principles and practices, 2nd Edition. Chap. 1.3

[2] S. Nemoto, Measurement of the refractive index of liquid using laser beam displacement, Applied

Optics, vol. 31, pp. 6690-6694 (1992)

[3] RefractiveIndex.info public database, Refractive index of $\mathrm{H} 2 \mathrm{O}, \mathrm{D} 2 \mathrm{O}$ (Water, heavy water, ice), https://refractiveindex.info/?shelf=main\&book=H2O, Accessed on May 1 (2019). 